\title{
Temporal Pattern/Episode Duration-based Classification of Atrial Fibrillation as Paroxysmal vs. Persistent: Is it time to develop a more integrated prognostic score to optimise management?
}

Matthew Hammond-Haley* MSc a, Rui Providencia* MD PhD b, Pier D. Lambiase PhD FRCP FHRS b,

*These authors contributed equally to this work

a Brighton and Sussex Medical School, Brighton, UK

b Bart's Heart Centre, Bart's Health NHS Trust, London, UK

c Institute of Cardiovascular Science, University College \& Barts Heart Centre, London, UK

Corresponding Author: Prof. Pier D. Lambiase, Institute of Cardiovascular Science, Paul O'Gorman Building, Huntley St, London WC1E 6BT.

p.lambiase@ucl.ac.uk

Tel: +442076796463.

PDL is supported by the National Institute for Health Research Biomedical Research Centre at University College London Hospitals NHS Foundation Trust and University College London. 


\section{Abstract}

Atrial fibrillation (AF) is a clinically heterogeneous arrhythmia that is currently classified according to the variety of temporal rhythm based manifestations observed clinically, including 'first detected episode', 'paroxysmal', 'persistent' or 'permanent' AF. Despite being widely adopted by the most recent consensus guidelines, the utility of this classification system to stratify patients into distinct and clinically useful groups that have different outcomes including mortality, morbidity, specific cardiovascular outcomes and quality of life, remains questionable. This is particularly true regarding the distinction between paroxysmal and persistent AF. Here, we demonstrate there is limited and conflicting evidence that this distinction is useful in predicting mortality, morbidity (including stroke and heart failure), symptom burden and quality of life, although there is convincing evidence that patients with paroxysmal AF have favourable outcomes following electrical cardioversion and have better ablation procedural outcomes. Risk stratification schemes including the $\mathrm{CHA}_{2} \mathrm{DS}_{2} \mathrm{VASc}$ score are widely used to help clinicians stratify patients at high risk of stroke, whilst a number of newer, potentially superior, competitors have also recently been developed. A range of parameters including left atrial (LA) size, LA morphology, LA fibrosis (assessed by MRI) LA appendage morphology and LA function (assessed by echo) have all been recently suggested to identify higher risk patients and may, perhaps in combination with validated risk stratification scores and emerging genetic data, provide clinicians with the information 
necessary to more accurately stratify patients to ensure better outcomes of specific AF treatments and prevent adverse events.

Key-words: arrhythmia; mortality; stroke; catheter ablation; cardioversion. 


\section{Introduction}

Atrial fibrillation (AF) is a common cardiac arrhythmia and a major public health problem currently estimated to affect $1-2 \%$ of the population in the developed world. Its incidence and prevalence is set to increase significantly as the population ages ${ }^{1}$. AF is strongly associated with ischaemic stroke ${ }^{2}$, increased allcause mortality ${ }^{3}$ and carries a substantial socio-economic burden ${ }^{45}$. Despite major advances in our understanding of the pathogenesis and natural history of $\mathrm{AF}$, the classification system currently used to categorize AF has remained relatively unchanged over recent decades. When used effectively, classifying disease into clinically discrete subtypes can be helpful in guiding management decisions as well as in selecting patients for inclusion in randomized control trials. However, the validity, reliability and clinical utility of the current temporal pattern based classification system of AF (Table 1), particularly into paroxysmal vs. persistent $\mathrm{AF}$, remains questionable ${ }^{6}$. This paper aims to examine the clinical utility of the current classification of $\mathrm{AF}$ into paroxysmal vs. persistent, and explore the alternative classification methods currently under investigation.

\section{Current AF classification}

Since the 2001 joint American Heart Association (AHA), American College of Cardiology (ACC) and European Society of Cardiology (ESC) consensus guidelines AF has been classified as a first detected episode, paroxysmal, persistent or permanent ${ }^{7}$. This forms the basis of the current classification system based on temporal rhythm patterns seen in AF and has become the most widely adopted 
classification system worldwide, still present in the most recent ACC/AHA ${ }^{8}$ and ESC/EHRA 9 guidelines. AF has previously been classified in a variety of ways, including the underlying pathology (such as myocardial infiltration or inflammation, atrial scar, atrial stretch, hormonal, thyroid disease, or idiopathic or 'lone $\mathrm{AF}^{\prime}$ ), ventricular rate (e.g. fast $\mathrm{AF}$ and slow $\mathrm{AF}$ ), and ECG features (e.g. high

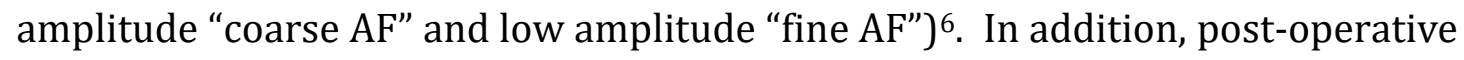
$\mathrm{AF}$, a common complication following cardiac surgery ${ }^{10}$, is often considered as a distinct clinical entity with differing treatment and prognosis, and is outside the scope of this report.

In patients with untreated or ineffectively treated AF, a proportion of patients will revert to sinus rhythm spontaneously whilst some patients will remain in $\mathrm{AF}$ permanently ${ }^{11}$. This clinical heterogeneity has been recognized for almost a century, with the terms "paroxysmal" and "chronic" used to described AF as early as the $1920 \mathrm{~s}^{12}$, and used widely throughout the $20^{\text {th }}$ century. Lewis was the first to formally recognize this phenomenon in 1922, stating that AF (then termed 'auricular fibrillation') "...was known to exist in two forms: in the form of short paroxysms, lasting a few hours or a few days, and much more often as a malady persisting until death. The view was held that if auricular fibrillation had lasted ten days, then it was a settled thing" and suggested that "...once established for a few weeks, the prospect of it's spontaneous passage is beyond reasonable explanation."12. This prompted the introduction of the term 'chronic AF', which was widely used throughout the $20^{\text {th }}$ century. 
However, given that patients can alternate between AF and sinus rhythm over many years, the use of "chronic" AF became unpopular as, despite AF being a chronic disease in such patients, they are not permanently ("chronically") in AF. Furthermore, with the wide application of electrical and pharmacological cardioversion, patients with previously "chronic" AF could be effectively returned to sinus rhythm, as cardioversion could be repeated whenever deemed appropriate ${ }^{13}$. Additionally, a significant proportion of patients with "paroxysmal $\mathrm{AF}^{\prime \prime}$ may progress to persistent $\mathrm{AF}$ (15\% in the first 12 months of follow up in one large prospective study of over 1200 paroxysmal AF patients ${ }^{14}$ ).

There is also marked clinical variability within paroxysmal AF, with some patients having very few short-lived episodes, whilst others have longer periods of AF and short periods of sinus rhythm. However, the cumulative time spent in AF, termed the 'AF density'15, has been difficult to accurately measure, given that most outpatient investigations provide only a 'snapshot' view of the heart's rhythm ${ }^{15}$, and even 24-hour Holter Monitor tests still underestimate AF density and overestimate success following ablation procedures ${ }^{15}$. Information from implantable cardiac devices (pacemakers and defibrillators), or implantable loop recorders can enable more precise quantification of AF burden if the sensing algorithms are optimised, and may provide insight into the development of AF over time ${ }^{16,17}$, but these methods are not applicable to the global population of $\mathrm{AF}$ patients. 
Early data on AF episode duration and thromboembolic risk were contradictory, but it is now almost accepted that patient-related risk factors included in current risk stratification schemes (e.g. $\mathrm{CHA}_{2} \mathrm{DS}_{2} \mathrm{VASc}$ ) are more important than AF type (paroxysmal or persistent). To date few studies have shown an association with the current AF classification and thromboembolic risk (Table 3).

Much of the scrutiny into the limitations of categorizing AF as either paroxysmal or chronic came in the 1990s. In 1995 Samuel Levy suggested a new classification system for paroxysmal AF that took into consideration an estimate of the frequency and duration of AF episodes, in order to better reflect risk. ${ }^{18}$. However, Levy's classification system only aimed to separate patients into different subtypes of paroxysmal AF based on risk, and therefore did not reflect the broad heterogeneity of all temporal patterns seen in AF. In 1998 Gallagher and Camm suggested an updated temporal rhythm based classification aiming to cover the majority of eventualities ${ }^{13}$, introducing the terms "persistent" and "permanent" alongside paroxysmal and first onset (acute) AF.

Paroxysmal AF was described as cases in which episodes of AF terminate spontaneously, and the authors decided not to subdivide this category further, as previously suggested ${ }^{18}$, arguing instead that the typical frequency and duration should be described for each individual patient, given the continuous nature of each variable. "Persistent AF" was used to describe AF which continues until a measure is taken to terminate it, whilst AF was described as "permanent" when all attempts to restore sinus rhythm had failed or when the patient and physician 
decide that no such attempt should be made ${ }^{13}$. These four categories form the basis of the classification system currently used by the ACC, AHA and ESC (EHRA), which was first endorsed in $2001^{7}$. The definitions currently used to classify AF by the ACC/AHA/ESC/EHRA and their evolution over time are shown in Tables 1 and 2 respectively.

\section{Evidence base for current definitions of paroxysmal vs. persistent AF}

Despite a wide adoption of this temporal rhythm based classification of AF, most probably because of its simplicity and clinical convenience, there has been a limited number of studies directly investigating whether this is clinically helpful in predicting differences in AF-related morbidity or mortality. This is particularly true when distinguishing between paroxysmal and persistent AF.

\section{Morbidity, stroke and heart failure}

Stroke is 3 to 5 times more common in patients with 'non-valvular' AF compared to those without electrophysiological abnormalities ${ }^{6,19,20}$, and a number of studies have looked at whether temporal rhythm based AF class can be used to further stratify patients at increased risk of stroke. Patients with 'valvular' AF, which has been defined as AF in the presence of either a bioprosthetic heart valve or mitral stenosis, is associated with a significantly increased risk of thrombo-embolic events, and is discussed in detail elsewhere ${ }^{21}$. 
During the early 1980s it was generally considered that only paroxysmal AF was strongly associated with thromboembolism ${ }^{22}$. However, it was then shown that a comparable risk of embolism was also present in 'chronic $\mathrm{AF}^{23}$, and that temporal rhythm based classification of AF does not predict risk of stroke $24,25,26,27,28$, but much of the data comes from before the standardization of the definitions currently used 29,30,31,32,33. Furthermore, a number of study limitations have been suggested to account for these findings, including imbalances in antithrombotic prescribing rates between groups in the GISSI-AF24 and ACTIVE-W ${ }^{27}$ studies $^{34}$. Later epidemiological studies then contradicted earlier findings, suggesting instead that more chronic forms of AF were in fact associated with a higher risk of thromboembolism than paroxysmal AF 35,36.

With the development of various thromboembolism risk-stratification schemes such as the $\mathrm{CHADS}_{2}{ }^{37}$ and the refined $\mathrm{CHA}_{2} \mathrm{DS}_{2}-\mathrm{VASc}^{38}$, it was then found that commonly established risk factors for thromboembolism such as previous stroke/TIA, patient age, hypertension and diabetes, were better predictors of outcome than temporal rhythm based classes of $\mathrm{AF}^{6,39}$. These scores allow a moderate estimation of the risk of stroke, and a number of recently developed risk scores aim to provide a more accurate tool for risk stratification. These include the ATRIA $^{40}$, Framingham ${ }^{41} \mathrm{R}_{2}$ CHADS (derived from the ROCKET-AF cohort ${ }^{42}$ ), ABCstroke (derived from ARISTOTLE ${ }^{43}$ ) and the ENGAGE-AF biomarker risk score ${ }^{44}$, all of which have been suggested to be more effective than previous scores in their preliminary validation cohorts. However, to date these risk scores only assess the risk of stroke and none are able to clarify different bio-phenotypes of patients with substrates of $\mathrm{AF}$, and therefore, they are likely to be unable to replace the 
paroxysmal/persistent AF classification for that purpose. Despite this, these scores are able to successfully discriminate between high and low risk patients, yet none rely on the temporal rhythm based AF class.

More recently, however, a study by Steinberg et. al, again demonstrated a significantly increased risk of stroke in patients with persistent compared with paroxysmal $\mathrm{AF}^{34}$. This finding was supported by the ARISTOTLE trial comparing the outcomes of apixaban vs. warfarin between AF subtypes, which showed a significantly lower risk of stroke or systemic embolism for paroxysmal AF than for persistent or permanent AF (HR: 0.70, 95\% CI $(0.51,0.93), \mathrm{P}=0.015)^{45}$. In addition to stroke, silent cerebral infarcts have also been recently shown to be more than twice as common in patients with AF than those without, but this association was independent of AF type (paroxysmal vs. persistent) ${ }^{46}$.

Similarly, congestive heart failure is considerably more common in patients with $\mathrm{AF}$, in part because they share similar risk factors, but also because one may directly predispose to the other ${ }^{47}$. Furthermore, the concomitant presence of both symptomatic heart failure (with reduced or preserved systolic function) and AF is associated with significantly worse cardiovascular morbidity and mortality ${ }^{48}$.

The strongest data to date, from the Euro Heart Survey, which prospectively followed a cohort of over 4000 patients with AF over 1 year, demonstrated similar rates of new-onset heart failure in patients with paroxysmal and persistent $\mathrm{AF}^{26}$. By contrast, the ARISTOTLE trial (which included almost 16,000 patients and therefore had more statistical power to show small differences) showed that 
patients with persistent $\mathrm{AF}$ were more likely to have a history of heart failure than patients with paroxysmal $\mathrm{AF}^{45}$.

Given the conflicting reports discussed, it is currently unclear whether the temporal rhythm based categories of paroxysmal and persistent AF are useful in predicting risk of stroke or heart failure.

\section{Mortality}

A number of studies have examined the relationship between class of AF and mortality. Overall, the data shows that patients with permanent AF have a worse prognosis than those with other patterns $26,49,50,51$, but there is an unclear difference between patients with paroxysmal vs. persistent $\mathrm{AF}^{26}$. Some studies have suggested a reduced mortality in persistent compared with paroxysmal $\mathrm{AF}^{49,52}$, whilst others have demonstrated a reduced all-cause mortality in paroxysmal AF compared with persistent AF ${ }^{34}$. These differences may be due to misclassifications of AF pattern, as again the majority of these studies did not strictly follow the definitions currently used to define paroxysmal and persistent $\mathrm{AF}$, cohorts with different risk profiles, or unaccounted confounding variables. Therefore, the ability of the paroxysmal and persistent AF classification to differentiate between two groups of patients with different prognosis also remains unclear.

\section{Symptoms and quality of life}

Improving quality of life (QoL) is becoming increasingly recognised as an important component of a comprehensive treatment plan as well as an outcome 
measure in studies investigating the efficacy of therapeutic interventions in AF53. QoL is impaired in the majority of patients with AF, largely due to the distress caused by symptom onset (including palpitations, chest pain, dizziness and heartfailure-like symptoms) ${ }^{53,54}$, and can be improved by both rate and rhythm control strategies ${ }^{55}$.

Investigation into QoL in AF in Spain showed that QoL varied significantly by clinical characteristics of the disease but there was no difference between paroxysmal, persistent and permanent $\mathrm{AF}$, except patients with permanent $\mathrm{AF}$ scored highest (better QoL) on psychological dimensions ${ }^{56}$. Better psychological QoL scores in more long-standing AF is perhaps because such patients, particularly with permanent AF, typically have more stable heart rate profiles with fewer paroxysmal changes and hence fewer symptomatic episodes over time, reducing the associated stress and anxiety ${ }^{53,57}$.

The Canadian Cardiovascular Society (CCS) developed a Severity of Atrial Fibrillation (CCS-SAF) in order to characterize patients' symptom severity and effect on wellbeing58. Patients were categorized by AF type (paroxysmal vs. persistent/permanent) and QoL was found to vary significantly by AF pattern, with patients with persistent or permanent AF reporting worse QoL than patients with paroxysmal AF58. By contrast a French study, again before the standardization of classification systems, showed that patients with paroxysmal AF (defined as "recurrent episodes of AF lasting $>2$ minutes and $<7$ days" and including patients with a first episode of AF lasting < 7days or cardioverted within 7 days) were significantly more symptomatic than patients with 'chronic AF' 
(defined as AF present for $>1$ month) ${ }^{29}$. These conflicting results may in part be confounded by the concomitant use of rate or rhythm control drugs that reduce adverse effects.

\section{Predicting outcomes following ablation}

Classifying $\mathrm{AF}$ as either paroxysmal or persistent successfully differentiates between two groups of patients with different outcomes in AF ablation. This is supported by convincing data from observational studies ${ }^{59}$ and large randomized controlled trials showing that catheter ablation is less successful for persistent AF than for paroxysmal AF59,60. Data from the Leipzig Heart Centre AF Ablation registry, which studied over 2000 patients also showed persistent AF to be an important predictor of AF recurrence at 12 months ${ }^{61}$. Indeed, data from Chao and colleagues show that long-term success rates after a single ablation procedure in non-paroxysmal AF may be as low as $25 \%$, and multiple procedures are often required 62

As such, a number of scoring systems aiming to predict the success of ablation, such as the APPLE63, BASE-AF2 ${ }^{64}$, and MB-LATER ${ }^{65}$ scores, include the temporal rhythm based classification of $\mathrm{AF}$, with persistent $\mathrm{AF}$ a predictor of poorer outcomes. Age, renal dysfunction, LA enlargement, BMI, duration of AF, current smoking status, left bundle branch block and male gender are among the other variables incorporated into these scores."

These findings are reflected by the current (2012) HRS/EHRA/ECAS expert consensus guidelines on catheter and surgical ablation of atrial fibrillation, which 
suggest that catheter ablation is "recommended" in paroxysmal AF (Class of Recommendation: I; level of evidence: A) and "reasonable" in persistent AF (Class of Recommendation: IIa; level of evidence: B) ${ }^{66}$. In contrast with the most recent ESC and AHA/ACC guidelines, this consensus provides slightly different definitions for paroxysmal and persistent AF (Table 2). Briefly, the authors suggest including patients with continuous AF who undergo cardioversion within 48 hours of $\mathrm{AF}$ onset as having paroxysmal $\mathrm{AF}$, and persistent $\mathrm{AF}$ if the cardioversion is performed more than 48 hours but less than one week after the onset of AF66.

\section{Is there a mechanistic basis for a symptom-based classification?}

It was suggested that the lower success rate of persistent AF compared with paroxysmal AF ablation may be explained by a mechanistic difference between the two forms, with paroxysmal AF considered to show a predominance of local triggers/drivers, particularly from the pulmonary veins (PVs), whilst in persistent AF re-entrant substrates (initially functional and then structural) predominate following electrical \& structural remodeling of the atria ${ }^{67}$. This was supported by early reports of significantly improved efficacy of pulmonary vein isolation in paroxysmal AF compared with persistent AF68.

In addition, structural remodeling of the left atrium has been shown to correlate with reduced electrogram amplitude in these areas ${ }^{69}$. In keeping with previous findings, more of these low voltage sites were seen in persistent AF, which may help individualize ablation strategies in such patients ${ }^{69}$. According to data from Marrouche and colleagues, the amount of atrial tissue fibrosis, estimated by 
delayed enhancement MRI, is independently associated with likelihood of recurrent arrhythmia ${ }^{70}$.

Recent investigations into catheter ablation in patients with persistent $\mathrm{AF}$ in the STAR-AF II trial ${ }^{59}$ showed that pulmonary vein isolation alone can achieve a successful outcome in approximately half of all patients with persistent AF, and that no additional benefit was obtained from additional linear or complex atrial fractionated potential ablation. However, these data are contested as many patients were in an early phase of persistent AF.

The failure of these trials to demonstrate the benefit of ablation beyond pulmonary vein isolation most probably reflects the heterogeneity of mechanisms involved, degree of atrial structural remodeling and limitations in current mapping technologies to define them, as well as the limitations of ablation as an appropriate strategy in all cases.

Whereas for paroxysmal AF pulmonary vein isolation can achieve a high rate of "cure" from AF relapse, things are more difficult to interpret in persistent AF. Approximately $50 \%$ of patients at 12 months do well with pulmonary vein isolation, suggesting that they present with pulmonary vein-dependent persistent AF. On the other hand, other patients presenting with relapse can represent one of two groups: (1) patients with involvement of other triggers or circuits outside the pulmonary veins, and which could not be effectively neutralized with the ablation technology (non-contact-force sensing radiofrequency), and strategies used (ablation of complex fractionated potentials acquired through the automatic 
NAV-X mapping algorithm, and linear ablation), or (2) patients with pulmonary vein-dependent persistent $A F$, whose veins reconnected following ablation.

Whilst there is considerable evidence to suggest a gradual evolution in the mechanisms driving AF over time, it still is unclear how the current classifications of AF into paroxysmal and persistent relate to this.

\section{Is the 7 day limit useful?}

$\mathrm{AF}$ is described as paroxysmal when lasting $<7$ days, and persistent or permanent when lasting $>7$ days (Table 1 ). This 7-day cutoff has been suggested to be useful in predicting the likelihood of spontaneous cardioversion or successful chemical or electrical cardioversion. Newer onset AF ( $<7$ days) is considered to have an increased chance of successful cardioversion than more longstanding (persistent) $\mathrm{AF}^{71}$. However, a range of studies have examined different cutoffs for recent-onset $\mathrm{AF}$, including $<24 \mathrm{~h}^{72,73},<72 \mathrm{~h}^{73}$ and $<2$ weeks ${ }^{72}$. All of these studies demonstrate that a shorter duration of $\mathrm{AF}$ is associated with increased chance of cardioversion. One study looking at the likelihood of spontaneous cardioversion within $72 \mathrm{~h}$ found presentation with symptoms of $<24 \mathrm{~h}$ duration was the best predictor of spontaneous cardioversion ${ }^{73}$.

In the development program of vernakalant, the 7-day cutoff was used to separate AF of short duration ( 3 hours to 7 days), and long duration ( 8 days to 45 days) ${ }^{74,75}$. No justification was given for the 7 day cutoff, but, interestingly vernakalant was more effective in cardioverting patients in the short AF duration group (over $50 \%$ success), than in the long AF duration group (less than $10 \%)^{74,75}$. 
Furthermore, chemical and electrical cardioversion have shown higher success rates when $\mathrm{AF}$ has existed for a shorter time. Chemical cardioversion by flecainide was shown to be significantly more successful in patients with an onset of AF $<24$ h compared with AF of longer duration ${ }^{76}$. Furthermore, amiodarone was shown to cardiovert $85 \%$ of patients with $\mathrm{AF}$ lasting < 1 year, compared to $57 \%$ in patients with $\mathrm{AF}$ lasting longer than 1 year ${ }^{77}$, and similar findings have shown that electrical cardioversion is also more successful in AF of shorter duration ${ }^{78}$.

Investigation of a goat model demonstrated that the length of artificially induced $\mathrm{AF}$ correlated with the duration of sustained $\mathrm{AF}$ following termination of the artificial $\mathrm{AF}^{79}$. Long-term sustained $\mathrm{AF}$ was a far more likely outcome following 2 weeks of sustained induced AF than following shorter episodes of induced AF, where spontaneous conversion to sinus rhythm was common ${ }^{79}$. Furthermore, the authors demonstrated a direct electrophysiological correlation between the atrial effective refractory period and the duration of the arrhythmia, demonstrating that AF promotes optimal electrophysiological conditions that favour further propagation of the arrhythmia. This prompted the widely accepted suggestion that "atrial fibrillation begets atrial fibrillation"79, and provides a electrophysiological explanation for the association between time spent in $\mathrm{AF}$ and likelihood of conversion to sinus rhythm.

In summary, whilst there is strong evidence to demonstrate an association between time spent in AF and likelihood of cardioversion, the 7-day distinction between paroxysmal and persistent AF is arbitrary. The ESC AF guidelines state 
that the 48 hour time point is clinically important in predicting the likelihood of spontaneous conversion to sinus rhythm, which falls significantly after 48 hours, and that anticoagulation should be considered after this cutoff ${ }^{80}$. There is strong evidence seems to support this ${ }^{73}$, which raises the question of why the 48 -hour mark is not used as a clinically significant differentiator between AF classes. This cutoff seems already to be slowly making its way into some of the expert consensus documents mentioned above ${ }^{66}$. Furthermore, the $24 \mathrm{~h}$ time point may be the most useful in predicting the probability of successful cardioversion.

\section{Future direction: towards improved AF classifications}

Clinical disease classification schemes should be able to discriminate between patients in order to inform clinical management decisions and predict the prognosis. As discussed, the current temporal rhythm based classification of AF is of limited or unclear value in this respect, and requires further investigation. By contrast, traditional risk stratification schemes such as CHA2DS2-VASc, particularly with the incorporation of cardiac biomarkers including troponins (raised in myocardial injury), BNP (raised in myocyte wall stress), amongst others $^{\mathbf{8 1}}$, may help to identify patients at high risk of thromboembolism ${ }^{\mathbf{4 3}, 44}$. In addition, a number of alternative methods of differentiating between subtypes of AF already exist, or are currently being investigated, including the emerging role AF genetic biomarkers ${ }^{\mathbf{8 2 , 8 3}}$, that may better distinguish between clinically relevant subgroups of patients with $\mathrm{AF}$. 
Does left atrial size by itself, independent of LA function, have any relevance?

Using data from the Framingham Heart Study, a study published in 1995 showed that, on multivariate analysis, left atrial enlargement remained a significant predictor of stroke in men, and death in both sexes ${ }^{84}$. Notably, this increased risk was not restricted to subjects with AF and eliminating such subjects did not significantly alter the association between atrial size and risk of stroke. This suggested that atrial size may be more important than atrial function (including temporal rhythm based class of AF) in predicting risk of stroke in all patients. Furthermore, recent sub-analysis of the ENGAGE-AF trial showed that increased left atrial size and higher filling pressures were associated with an increased risk of death, but not thromboembolism ${ }^{85}$.

Recent investigations in this area suggest that the geometry of the left atrium, rather than size alone, may be a better predictor of thromboembolic risk $^{86}$. Incorporating this information, termed 'left atrial sphericity', into the CHADS 2 and $\mathrm{CHA}_{2} \mathrm{DS}_{2}$-VASc risk scores significantly improves their performance ${ }^{86}$. Furthermore, it has also been suggested that left atrial appendage (one of the main sources of cardiac thrombus formation) morphology may also be associated with embolic risk, with one study showing that patients with a normal 'chicken wing' shaped left atrial appendage had a considerably lower risk of thromboembolism ${ }^{87}$. This finding was also supported by a recent meta-analysis including a total of 8 studies and over 2,500 patients ${ }^{88}$, suggesting that left atrial appendage morphology may indeed offer useful information in predicting risk of thromboembolism and guiding anticoagulation management. This is particularly 
relevant in determining risk for patients with a $\mathrm{CHA}_{2} \mathrm{DS}_{2}$-VASc score of 1 , where the benefits and risks of anticoagulation are more finely balanced versus higher $\mathrm{CHA}_{2} \mathrm{DS}_{2}$-VASc scores.

\section{Can measures of left atrial function as assessed by echo be of use?}

Certain echocardiogram findings reflecting left atrial function (like low velocities in the in the left atrial appendage, spontaneous echocardiographic contrast and presence of left atrial thrombus on transoesophageal echocardiogram and left atrial strain and strain rate analysis ${ }^{89}$, which can be performed through transthoracic echocardiogram) in $\mathrm{AF}$ are associated with an increased risk of thromboembolic disease ${ }^{90}$, and may be helpful in refining and improving the accuracy of current risk stratification schemes such as $\mathrm{CHA}_{2} \mathrm{DS}_{2}-\mathrm{VASc}^{91}$. Echocardiography is a widely used and relatively cheap imaging modality making this a very promising possibility. If these techniques provide a reliable surrogate of fibrosis, they would be a likely candidate. However a number of unsolved questions exist: the transoesophageal echocardiogram is an invasive test and therefore cannot be used in the global AF population; assessment of left atrial strain and strain rate is difficult and operator-dependent, requiring expertise, and no consensus exists regarding the best methodology to assess it $^{92}$.

\section{Fibrosis assessed by MRI}

Some authors have suggested an important role for fibrotic atrial structural remodeling as a potential cause of abnormal atrial activation and hence initiation and propagation of $\mathrm{AF}$, with the degree of fibrosis varying widely among patients $93,94,70$. Patients with extensive fibrosis may represent a phenotype of 
advanced and irreversible atrial myopathy, while patients without fibrosis can represent either forms of purely electrical disease (i.e. "atrial chanelopathy"), or patients in very early stages of the progression of a fibrotic myopathy.

Fibrosis of the left atrium has been suggested to be a useful predictor for success of rhythm control strategies including catheter ablation ${ }^{95,79}$. Hence, detecting the extent of atrial remodeling, which can be effectively achieved by delayed enhancement magnetic resonance imaging (MRI), may play an important role in differentiating between patients with AF to help predict treatment response and guide prognosis. This "UTAH classification" has been associated with outcomes of AF ablation ${ }^{96}$.

Preliminary data suggest that extension of left atrial fibrosis measured through MRI may be associated with prior history of stroke in AF individuals ${ }^{97}$, and with the presence of a more pro-thrombotic milieu in the left atrium ${ }^{98}$. However, the association of left atrial fibrosis with major outcomes like stroke, heart failure and mortality remains to be proven.

\section{Scoring systems for predicting progression to persistent AF}

A significant proportion of patients with paroxysmal AF will progress to persistent $\mathrm{AF}$, and these patients often have worse outcomes than patients with AF who demonstrate no progression ${ }^{14}$. The HATCH score was developed to predict progression, based on a number of independent predictors of AF progression identified from multivariate analysis of a cohort of 1,219 patients with paroxysmal AF (15\% of which progressed to more sustained forms over a 12 month-interval), 
including heart failure, previous transient ischaemic attack or stroke, hypertension and chronic obstructive pulmonary disease ${ }^{14}$. The HATCH score may be a useful tool for selecting patients for intensified ECG monitoring ${ }^{99}$, and may also be useful in stroke risk assessment ${ }^{99}$, but there is currently limited data to support this. Recent investigation into the progression of paroxysmal to persistent AF in patients awaiting ablation showed that the HATCH score was a poor predictor of progression, and multivariate analysis suggested a left atrial diameter of $>45 \mathrm{~mm}$ and heart failure were the strongest, independent predictors of $\mathrm{AF}$ $\operatorname{progression}^{100}$.

\section{Discussion}

The current temporal rhythm based classification of AF provides a simple and reproducible way of describing the broad range of clinical patterns seen. It has the advantage of standardizing the terminology used and has led to the gradual disuse of vague terms and misleading terms such as 'chronic' AF6. However, as discussed, this strategy has a number of important limitations.

Firstly, there is little evidence to show any clinical utility in differentiating between paroxysmal and persistent AF in terms of morbidity, mortality, and quality of life. Furthermore, despite mechanistic differences between paroxysmal and persistent AF the distinction does not support different ablation strategies, following the results of the recent STAR-II AF trial ${ }^{59}$, and the 7 day cut-off which forms the basis of these terms appears to be arbitrary. Alternative concepts like early persistent $\mathrm{AF}^{101}$ and persistent $\mathrm{AF}$ from the onset ${ }^{102}$, are currently being 
introduced in routine clinical practice and may contribute to further identify different groups of patients with different pathophysiologic bases and likely to have a different prognosis, but can also lead to and aggravate some of the confusion on classifying patients who present on the borderline between paroxysmal and persistent AF ablation, combining features of both forms.

In addition, there is a lack of standardization in multiple aspects of $\mathrm{AF}$ management, including diagnosis, ECG findings and the methods used to monitor the duration and extent of heart rhythm and rate. This is further compounded by continuously evolving AF treatments including new drugs, techniques, procedures and devices, with varying patient selection criteria (including the concomitant use of drugs to control rate, rhythm and other agents known to affect cardiovascular outcomes) and outcomes measured in RCTs to date. As a result, there is a lack of RCT data using standardized definitions and approaches for diagnosis, monitoring, treatment and outcomes, which limits the evaluation and refinement of current AF nomenclature needed to improve patient outcomes.

A number of alternative risk stratification methods are currently being investigated. If after thorough investigation, including pilot studies and larger RCTs, such methods remain promising and feasible, these approaches may be combined with traditional risk stratification systems to provide a more individualized, integrated and comprehensive stratgey to determine which patients are more likely to respond favourably to particular therapeutic modalities. This will only be possible by harvesting high quality data from 
previous studies as well as appropriately designed, prospective trials following the standardized categorization, nomenclature and management of AF.

In addition, recent attempts have been made to more broadly categorize atrial pathologies into discrete cohorts based on underlying histopathology as part of the spectrum of 'atrial cardiomyopathies', defined by a recent expert consensus as "Any complex of structural, architectural, contractile or electrophysiological changes affecting the atria with the potential to produce clinically-relevant manifestations"103. This 'EHRAS' classification system may be a more useful way of categorizing patients with $\mathrm{AF}$, enable individualized management and improve outcomes, but still requires validation ${ }^{103}$.

\section{Conclusion}

Apart from being a simple division predicting success of cardioversion and pulmonary vein isolation in early onset $\mathrm{AF}$, the current classification of AF into paroxysmal / persistent has so far failed to demonstrate a robust association with symptom severity, quality of life and cardiovascular outcomes, The cardiology community should continue to develop more clinically relevant classification schemes that better predict outcomes and support evidence based management decisions.

Conflict of interest: PDL receives speaker fees from Boston Scientfic and educational grants from Boston Scientific, Medtronic, St Jude Medical. 
1. Schnabel RB, Yin X, Gona P, Larson MG, Beiser AS, McManus DD, et al. 50 year trends in atrial fibrillation prevalence, incidence, risk factors, and mortality in the Framingham Heart Study: a cohort study. The Lancet 2015;386:15462.

2. Wolf PA, Dawber TR, Thomas HE, Kannel WB. Epidemiologic assessment of chronic atrial fibrillation and risk of stroke: the Framingham study. Neurology 1978;28:973-7.

3. Benjamin EJ, Wolf PA, D’Agostino RB, Silbershatz H, Kannel WB, Levy D. Impact of atrial fibrillation on the risk of death: the Framingham Heart Study. Circulation 1998;98:946-52.

4. Stewart S, Murphy N, Walker A, McGuire A, McMurray JJV. Cost of an emerging epidemic: an economic analysis of atrial fibrillation in the UK. Heart 2004;90:286-92.

5. Kim MH, Johnston SS, Chu B-C, Dalal MR, Schulman KL. Estimation of total incremental health care costs in patients with atrial fibrillation in the United States. Circ Cardiovasc Qual Outcomes 2011;4:313-20.

6. Lubitz SA, Benjamin EJ, Ruskin JN, Fuster V, Ellinor PT. Challenges in the classification of atrial fibrillation. Nat Rev Cardiol 2010;7:451-60.

7. Fuster V, Rydén LE, Asinger RW, Cannom DS, Crijns HJ, Frye RL, et al. ACC/AHA/ESC guidelines for the management of patients with atrial fibrillation: executive summaryA Report of the American College of Cardiology/ American Heart Association Task Force on Practice Guidelines and the European Society of Cardiology Committee for Practice Guidelines and Policy Conferences (Committee to Develop Guidelines for the Management of Patients With Atrial Fibrillation) Developed in Collaboration With the North American Society of Pacing and Electrophysiology333. J Am Coll Cardiol 2001;38:1231-65.

8. January CT, Wann LS, Alpert JS, Calkins H, Cigarroa JE, Cleveland J Joseph C., et al. 2014 AHA/ACC/HRS Guideline for the Management of Patients With Atrial FibrillationA Report of the American College of Cardiology/American Heart Association Task Force on Practice Guidelines and the Heart Rhythm Society. J Am Coll Cardiol 2014;64:e1-76.

9. Kirchhof P, Benussi S, Kotecha D, Ahlsson A, Atar D, Casadei B, et al. 2016 ESC Guidelines for the management of atrial fibrillation developed in collaboration with EACTS The Task Force for the management of atrial fibrillation of the European Society of Cardiology (ESC) Developed with the special contribution of the European Heart Rhythm Association (EHRA) of the ESCEndorsed by the European Stroke Organisation (ESO). Eur J Cardiothorac Surg 2016; ezw313. 
10. Echahidi N, Pibarot P, O’Hara G, Mathieu P. Mechanisms, Prevention, and Treatment of Atrial Fibrillation After Cardiac Surgery. J Am Coll Cardiol 2008;51:793-801.

11. Kerr CR, Humphries KH, Talajic M, Klein GJ, Connolly SJ, Green M, et al. Progression to chronic atrial fibrillation after the initial diagnosis of paroxysmal atrial fibrillation: results from the Canadian Registry of Atrial Fibrillation. Am Heart J 2005;149:489-96.

12. Lewis T. The value of quinidine in cases of auricular fibrillation and methods of studying the clinical reaction. Am J Med Sci 1922;163:781-94.

13. Gallagher MM, Camm J. Classification of atrial fibrillation. Am J Cardiol 1998;82:18N - 28N.

14. Vos CB de, Pisters R, Nieuwlaat R, Prins MH, Tieleman RG, Coelen R-JS, et al. Progression from paroxysmal to persistent atrial fibrillation: clinical correlates and prognosis. J Am Coll Cardiol 2010;55:725-31.

15. Charitos EI, Stierle U, Ziegler PD, Baldewig M, Robinson DR, Sievers H-H, et al. A comprehensive evaluation of rhythm monitoring strategies for the detection of atrial fibrillation recurrence: insights from 647 continuously monitored patients and implications for monitoring after therapeutic interventions. Circulation 2012;CIRCULATIONAHA - 112.

16. Hindricks G, Pokushalov E, Urban L, Taborsky M, Kuck K-H, Lebedev D, et al. Performance of a New Leadless Implantable Cardiac Monitor in Detecting and Quantifying Atrial Fibrillation Results of the XPECT TrialCLINICAL PERSPECTIVE. Circ Arrhythm Electrophysiol 2010;3:141-7.

17. Sugihara C, Veasey R, Freemantle N, Podd S, Furniss S, Sulke N. The development of AF over time in patients with permanent pacemakers: objective assessment with pacemaker diagnostics demonstrates distinct patterns of AF. EP Eur 2015;17:864-70.

18. Levy S, Novella P, Ricard P, Paganelli F. Paroxysmal A trial Fibrillation: J Cardiovasc Electrophysiol 1995;6:69-74.

19. Stewart S, Hart CL, Hole DJ, McMurray JJV. A population-based study of the long-term risks associated with atrial fibrillation: 20-year follow-up of the Renfrew/Paisley study. Am J Med 2002;113:359-64.

20. Wolf PA, Abbott RD, Kannel WB. Atrial fibrillation as an independent risk factor for stroke: the Framingham Study. Stroke J Cereb Circ 1991;22:983-8.

21. Caterina RD, Camm AJ. What is 'valvular' atrial fibrillation? A reappraisal. Eur Heart J 2014;35:3328-35.

22. Petersen P, Godtfredsen J. Embolic complications in paroxysmal atrial fibrillation. Stroke J Cereb Circ 1986;17:622-6. 
23. Wolf PA, Kannel WB, McGee DL, Meeks SL, Bharucha NE, McNamara PM. Duration of atrial fibrillation and imminence of stroke: the Framingham study. Stroke J Cereb Circ 1983;14:664-7.

24. Disertori M, Franzosi MG, Barlera S, Cosmi F, Quintarelli S, Favero C, et al. Thromboembolic event rate in paroxysmal and persistent atrial fibrillation: data from the GISSI-AF trial. BMC Cardiovasc Disord 2013;13:28.

25. Hart RG, Pearce LA, Rothbart RM, McAnulty JH, Asinger RW, Halperin JL. Stroke with intermittent atrial fibrillation: incidence and predictors during aspirin therapy. Stroke Prevention in Atrial Fibrillation Investigators. J Am Coll Cardiol 2000;35:183-7.

26. Nieuwlaat R, Prins MH, Le Heuzey J-Y, Vardas PE, Aliot E, Santini M, et al. Prognosis, disease progression, and treatment of atrial fibrillation patients during 1 year: follow-up of the Euro Heart Survey on atrial fibrillation. Eur Heart J 2008;29:1181-9.

27. Hohnloser SH, Pajitnev D, Pogue J, Healey JS, Pfeffer MA, Yusuf S, et al. Incidence of stroke in paroxysmal versus sustained atrial fibrillation in patients taking oral anticoagulation or combined antiplatelet therapy: an ACTIVE W Substudy. J Am Coll Cardiol 2007;50:2156-61.

28. Friberg L, Hammar N, Rosenqvist M. Stroke in paroxysmal atrial fibrillation: report from the Stockholm Cohort of Atrial Fibrillation. Eur Heart J 2010;31:967-75.

29. Lévy S, Maarek M, Coumel P, Guize L, Lekieffre J, Medvedowsky JL, et al. Characterization of different subsets of atrial fibrillation in general practice in France: the ALFA study. The College of French Cardiologists. Circulation 1999;99:3028-35.

30. Sage JI, Van Uitert RL. Risk of recurrent stroke in patients with atrial fibrillation and non-valvular heart disease. Stroke 1983;14:537-40.

31. Roy D, Marchand E, Gagné P, Chabot M, Cartier R. Usefulness of anticoagulant therapy in the prevention of embolic complications of atrial fibrillation. $\mathrm{Am}$ Heart J 1986;112:1039-43.

32. Moulton AW, Singer DE, Haas JS. Risk factors for stroke in patients with nonrheumatic atrial fibrillation: a case-control study. Am J Med 1991;91:156-61.

33. Risk factors for stroke and efficacy of antithrombotic therapy in atrial fibrillation. Analysis of pooled data from five randomized controlled trials. Arch Intern Med 1994;154:1449-57.

34. Steinberg BA, Hellkamp AS, Lokhnygina Y, Patel MR, Breithardt G, Hankey GJ, et al. Higher risk of death and stroke in patients with persistent vs. paroxysmal atrial fibrillation: results from the ROCKET-AF Trial. Eur Heart J 2014; ehu359. 
35. Kannel WB, Abbott RD, Savage DD, McNamara PM. Coronary heart disease and atrial fibrillation: the Framingham Study. Am Heart J 1983;106:389-96.

36. Treseder AS, Sastry BS, Thomas TP, Yates MA, Pathy MS. Atrial fibrillation and stroke in elderly hospitalized patients. Age Ageing 1986;15:89-92.

37. Gage BF, Waterman AD, Shannon W, Boechler M, Rich MW, Radford MJ. Validation of clinical classification schemes for predicting stroke: results from the National Registry of Atrial Fibrillation. JAMA 2001;285:2864-70.

38. Lip GYH, Nieuwlaat R, Pisters R, Lane DA, Crijns HJGM. Refining clinical risk stratification for predicting stroke and thromboembolism in atrial fibrillation using a novel risk factor-based approach: the euro heart survey on atrial fibrillation. Chest 2010;137:263-72.

39. Group SR in AFW. Comparison of 12 Risk Stratification Schemes to Predict Stroke in Patients With Nonvalvular Atrial Fibrillation. Stroke 2008;39:1901-10.

40. Aspberg S, Chang Y, Atterman A, Bottai M, Go AS, Singer DE. Comparison of the ATRIA, CHADS2, and CHA2DS2-VASc stroke risk scores in predicting ischaemic stroke in a large Swedish cohort of patients with atrial fibrillation. Eur Heart J 2016;ehw077.

41. Wang TJ, Massaro JM, Levy D, Vasan RS, Wolf PA, D’Agostino RB, et al. A risk score for predicting stroke or death in individuals with new-onset atrial fibrillation in the community: the Framingham Heart Study. JAMA 2003;290:1049-56.

42. Piccini JP, Stevens SR, Chang Y, Singer DE, Lokhnygina Y, Go AS, et al. Renal dysfunction as a predictor of stroke and systemic embolism in patients with nonvalvular atrial fibrillation: validation of the R(2)CHADS(2) index in the ROCKET AF (Rivaroxaban Once-daily, oral, direct factor Xa inhibition Compared with vitamin $\mathrm{K}$ antagonism for prevention of stroke and Embolism Trial in Atrial Fibrillation) and ATRIA (AnTicoagulation and Risk factors In Atrial fibrillation) study cohorts. Circulation 2013;127:224-32.

43. Hijazi Z, Lindbäck J, Alexander JH, Hanna M, Held C, Hylek EM, et al. The ABC (age, biomarkers, clinical history) stroke risk score: a biomarker-based risk score for predicting stroke in atrial fibrillation. Eur Heart J 2016;37:158290 .

44. Ruff CT, Giugliano RP, Braunwald E, Murphy SA, Brown K, Jarolim P, et al. Cardiovascular biomarker score and clinical outcomes in patients with atrial fibrillation enrolled in the ENGAGE AF-TIMI 48 trial. EUROPEAN HEART JOURNAL OXFORD UNIV PRESS GREAT CLARENDON ST, OXFORD OX2 6DP, ENGLAND; 2014. p. 440-440.

45. Al-Khatib SM, Thomas L, Wallentin L, Lopes RD, Gersh B, Garcia D, et al. Outcomes of apixaban vs. warfarin by type and duration of atrial fibrillation: results from the ARISTOTLE trial. Eur Heart J 2013;34:2464-71. 
46. Kalantarian S, Ay H, Gollub RL, Lee H, Retzepi K, Mansour M, et al. Association Between Atrial Fibrillation and Silent Cerebral InfarctionsA Systematic Review and Meta-analysisAtrial Fibrillation and Silent Cerebral Infarctions. Ann Intern Med 2014;161:650-8.

47. Wang TJ, Larson MG, Levy D, Vasan RS, Leip EP, Wolf PA, et al. Temporal Relations of Atrial Fibrillation and Congestive Heart Failure and Their Joint Influence on Mortality The Framingham Heart Study. Circulation 2003;107:2920-5.

48. Olsson LG, Swedberg K, Ducharme A, Granger CB, Michelson EL, McMurray JJV, et al. Atrial Fibrillation and Risk of Clinical Events in Chronic Heart Failure With and Without Left Ventricular Systolic DysfunctionResults From the Candesartan in Heart failure-Assessment of Reduction in Mortality and morbidity (CHARM) Program. J Am Coll Cardiol 2006;47:1997-2004.

49. Keating RJ, Gersh BJ, Hodge DO, Weivoda PL, Patel PJ, Hammill SC, et al. Effect of Atrial Fibrillation Pattern on Survival in a Community-Based Cohort. Am J Cardiol 2005;96:1420-4.

50. Ruigómez A, Johansson S, Wallander M-A, García Rodríguez LA. Predictors and prognosis of paroxysmal atrial fibrillation in general practice in the UK. BMC Cardiovasc Disord 2005;5:1-10.

51. Kannel WB, Abbott RD, Savage DD, McNamara PM. Epidemiologic features of chronic atrial fibrillation: the Framingham study. $N$ Engl J Med 1982;306:1018-22.

52. Friberg L, Hammar N, Pettersson H, Rosenqvist M. Increased mortality in paroxysmal atrial fibrillation: report from the Stockholm Cohort-Study of Atrial Fibrillation (SCAF). Eur Heart J 2007;

53. Aliot E, Botto GL, Crijns HJ, Kirchhof P. Quality of life in patients with atrial fibrillation: how to assess it and how to improve it. Europace 2014;eut369.

54. European Heart Rhythm Association, European Association for CardioThoracic Surgery, Camm AJ, Kirchhof P, Lip GYH, Schotten U, et al. Guidelines for the management of atrial fibrillation: the Task Force for the Management of Atrial Fibrillation of the European Society of Cardiology (ESC). Eur Eur Pacing Arrhythm Card Electrophysiol J Work Groups Card Pacing Arrhythm Card Cell Electrophysiol Eur Soc Cardiol 2010;12:1360420.

55. Thrall G, Lane D, Carroll D, Lip GYH. Quality of life in patients with atrial fibrillation: a systematic review. Am J Med 2006;119:448.e1-19.

56. Peinado R, Arribas F, Ormaetxe JM, Badía X. Variation in quality of life with type of atrial fibrillation. Rev Esp Cardiol 2010;63:1402-9.

57. Thrall G, Lip GYH, Carroll D, Lane D. Depression, anxiety, and quality of life in patients with atrial fibrillation. Chest 2007;132:1259-64. 
58. Dorian P, Cvitkovic SS, Kerr CR, Crystal E, Gillis AM, Guerra PG, et al. A novel, simple scale for assessing the symptom severity of atrial fibrillation at the bedside: the CCS-SAF scale. Can J Cardiol 2006;22:383-6.

59. Verma A, Jiang C, Betts TR, Chen J, Deisenhofer I, Mantovan R, et al. Approaches to Catheter Ablation for Persistent Atrial Fibrillation. $N$ Engl J Med 2015;372:1812-22.

60. Kuck K-H, Brugada J, Fürnkranz A, Metzner A, Ouyang F, Chun KRJ, et al. Cryoballoon or Radiofrequency Ablation for Paroxysmal Atrial Fibrillation. N Engl J Med 2016;0:null.

61. Kornej J, Hindricks G, Kosiuk J, Arya A, Sommer P, Husser D, et al. Comparison of CHADS2, R2CHADS2, and CHA2DS2-VASc Scores for the Prediction of Rhythm Outcomes After Catheter Ablation of Atrial FibrillationCLINICAL PERSPECTIVE. Circ Arrhythm Electrophysiol 2014;7:281-7.

62. Chao T-F, Tsao H-M, Lin Y-J, Tsai C-F, Lin W-S, Chang S-L, et al. Clinical Outcome of Catheter Ablation in Patients With Nonparoxysmal Atrial FibrillationClinical Perspective. Circ Arrhythm Electrophysiol 2012;5:51420.

63. Kornej J, Hindricks G, Shoemaker MB, Husser D, Arya A, Sommer P, et al. The APPLE score: a novel and simple score for the prediction of rhythm outcomes after catheter ablation of atrial fibrillation. Clin Res Cardiol OffJ Ger Card Soc 2015;104:871-6.

64. Canpolat U, Aytemir K, Yorgun H, Şahiner L, Kaya EB, Oto A. A proposal for a new scoring system in the prediction of catheter ablation outcomes: promising results from the Turkish Cryoablation Registry. Int J Cardiol 2013;169:201-6.

65. Mujović N, Marinković M, Marković N, Shantsila A, Lip GYH, Potpara TS. Prediction of very late arrhythmia recurrence after radiofrequency catheter ablation of atrial fibrillation: The MB-LATER clinical score. Sci Rep 2017;7:40828.

66. Calkins H, Kuck KH, Cappato R, Brugada J, Camm AJ, Chen S-A, et al. 2012 HRS/EHRA/ECAS Expert Consensus Statement on Catheter and Surgical Ablation of Atrial Fibrillation: Recommendations for Patient Selection, Procedural Techniques, Patient Management and Follow-up, Definitions, Endpoints, and Research Trial Design. Europace 2012;14:528-606.

67. Iwasaki Y, Nishida K, Kato T, Nattel S. Atrial Fibrillation Pathophysiology Implications for Management. Circulation 2011;124:2264-74.

68. Oral H, Knight BP, Tada H, Özaydın M, Chugh A, Hassan S, et al. Pulmonary Vein Isolation for Paroxysmal and Persistent Atrial Fibrillation. Circulation 2002;105:1077-81. 
69. Rolf S, Kircher S, Arya A, Eitel C, Sommer P, Richter S, et al. Tailored Atrial Substrate Modification Based on Low-Voltage Areas in Catheter Ablation of Atrial FibrillationCLINICAL PERSPECTIVE. Circ Arrhythm Electrophysiol 2014;7:825-33.

70. Marrouche NF, Wilber D, Hindricks G, et al. Association of atrial tissue fibrosis identified by delayed enhancement mri and atrial fibrillation catheter ablation: The decaaf study. JAMA 2014;311:498-506.

71. FALK RH, KNOWLTON AA, BERNARD SA, GOTLIEB NE, BATTINELLI NJ. Digoxin for Converting Recent-Onset Atrial Fibrillation to Sinus RhythmA Randomized, Double-Blinded Trial. Ann Intern Med 1987;106:503-6.

72. Weiner P, Ganam R, Ganem R, Zidan F, Rabner M. Clinical course of recentonset atrial fibrillation treated with oral propafenone. Chest 1994;105:1013-6.

73. Danias PG, Caulfield TA, Weigner MJ, Silverman DI, Manning WJ. Likelihood of Spontaneous Conversion of Atrial Fibrillation to Sinus Rhythm. J Am Coll Cardiol 1998;31:588-92.

74. Roy D, Pratt CM, Torp-Pedersen C, Wyse DG, Toft E, Juul-Moller S, et al. Vernakalant Hydrochloride for Rapid Conversion of Atrial Fibrillation. Circulation 2008;117:1518-25.

75. Pratt CM, Roy D, Torp-Pedersen C, Wyse DG, Toft E, Juul-Moller S, et al. Usefulness of Vernakalant Hydrochloride Injection for Rapid Conversion of Atrial Fibrillation. Am J Cardiol 2010;106:1277-83.

76. Crijns HJGM, Wijk LMV, Gilst WHV, Kingma JH, Gelder ICV, Lie KI. Acute conversion of atrial fibrillation to sinus rhythm: clinical efficacy of flecainide acetate. Comparison of two regimens. Eur Heart J 1988;9:634-8.

77. Gold RL, Haffajee CI, Charos G, Sloan K, Baker S, Alpert JS. Amiodarone for refractory atrial fibrillation. Am J Cardiol 1986;57:124-7.

78. Grönberg T, Hartikainen JEK, Nuotio I, Biancari F, Vasankari T, Nikkinen M, et al. Can we predict the failure of electrical cardioversion of acute atrial fibrillation? The FinCV study. Pacing Clin Electrophysiol PACE 2015;38:36875.

79. Wijffels MC, Kirchhof CJ, Dorland R, Allessie MA. Atrial fibrillation begets atrial fibrillation. A study in awake chronically instrumented goats. Circulation 1995;92:1954-68.

80. Members AF, Camm AJ, Lip GYH, Caterina RD, Savelieva I, Atar D, et al. 2012 focused update of the ESC Guidelines for the management of atrial fibrillation. Europace 2012;14:1385-413.

81. Hijazi Z, Oldgren J, Siegbahn A, Granger CB, Wallentin L. Biomarkers in atrial fibrillation: a clinical review. Eur Heart J 2013;34:1475-80. 
82. Lubitz SA, Yin X, Lin H, Kolek M, Smith JG, Trompet S, et al. Genetic Risk Prediction of Atrial Fibrillation. Circulation 2016;CIRCULATIONAHA.116.024143.

83. Christophersen IE, Ellinor PT. Genetics of atrial fibrillation: from families to genomes. J Hum Genet 2016;61:61-70.

84. Benjamin EJ, D’Agostino RB, Belanger AJ, Wolf PA, Levy D. Left Atrial Size and the Risk of Stroke and Death. Circulation 1995;92:835-41.

85. Gupta DK, Giugliano RP, Ruff CT, Claggett B, Murphy S, Antman E, et al. The Prognostic Significance of Cardiac Structure and Function in Atrial Fibrillation: The ENGAGE AF-TIMI 48 Echocardiographic Substudy. J Am Soc Echocardiogr 2016;29:537-44.

86. Bisbal F, Gómez-Pulido F, Cabanas-Grandío P, Akoum N, Calvo M, Andreu D, et al. Left Atrial Geometry Improves Risk Prediction of Thromboembolic Events in Patients With Atrial Fibrillation. J Cardiovasc Electrophysiol 2016;n/a - n/a.

87. Di Biase L, Santangeli P, Anselmino M, Mohanty P, Salvetti I, Gili S, et al. Does the left atrial appendage morphology correlate with the risk of stroke in patients with atrial fibrillation? Results from a multicenter study. J Am Coll Cardiol 2012;60:531-8.

88. Lupercio F, Diaz JC, Spinetto PV, Briceno D, Romero J, Gonzalez S, et al. LEFT ATRIAL APPENDAGE MORPHOLOGY AND STROKE RISK IN PATIENTS WITH ATRIAL FIBRILLATION: A META-ANALYSIS. J Am Coll Cardiol 2016;67:713-713.

89. Obokata M, Negishi K, Kurosawa K, Tateno R, Tange S, Arai M, et al. Left atrial strain provides incremental value for embolism risk stratification over $\mathrm{CHA}_{2} \mathrm{DS}_{2}$-VASc score and indicates prognostic impact in patients with atrial fibrillation. J Am Soc Echocardiogr Off Publ Am Soc Echocardiogr 2014;27:709-16.e4.

90. Dinh T, Baur LHB, Pisters R, Kamp O, Verheugt FWA, Smeets JLRM, et al. Aspirin versus vitamin $\mathrm{K}$ antagonist treatment guided by transoesophageal echocardiography in patients with atrial fibrillation: a pilot study. Heart $\mathrm{Br}$ Card Soc 2014;100:563-8.

91. Providência R, Botelho A, Trigo J, Quintal N, Nascimento J, Mota P, et al. Possible refinement of clinical thromboembolism assessment in patients with atrial fibrillation using echocardiographic parameters. Eur Eur Pacing Arrhythm Card Electrophysiol J Work Groups Card Pacing Arrhythm Card Cell Electrophysiol Eur Soc Cardiol 2012;14:36-45.

92. Providência R, Trigo J, Paiva L, Barra S. The role of echocardiography in thromboembolic risk assessment of patients with nonvalvular atrial fibrillation. J Am Soc Echocardiogr Off Publ Am Soc Echocardiogr 2013;26:801-12. 
93. Kottkamp H, Bender R, Berg J. Catheter Ablation of Atrial FibrillationHow to Modify the Substrate? J Am Coll Cardiol 2015;65:196-206.

94. Allessie M, Ausma J, Schotten U. Electrical, contractile and structural remodeling during atrial fibrillation. Cardiovasc Res 2002;54:230-46.

95. Morillo CA, Klein GJ, Jones DL, Guiraudon CM. Chronic rapid atrial pacing. Structural, functional, and electrophysiological characteristics of a new model of sustained atrial fibrillation. Circulation 1995;91:1588-95.

96. Oakes RS, Badger TJ, Kholmovski EG, Akoum N, Burgon NS, Fish EN, et al. Detection and Quantification of Left Atrial Structural Remodeling With Delayed-Enhancement Magnetic Resonance Imaging in Patients With Atrial Fibrillation. Circulation 2009;119:1758-67.

97. Daccarett M, Badger TJ, Akoum N, Burgon NS, Mahnkopf C, Vergara G, et al. Association of left atrial fibrosis detected by delayed-enhancement magnetic resonance imaging and the risk of stroke in patients with atrial fibrillation. J Am Coll Cardiol 2011;57:831-8.

98. Akoum N, Fernandez G, Wilson B, Mcgann C, Kholmovski E, Marrouche N. Association of Atrial Fibrosis Quantified Using LGE-MRI with Atrial Appendage Thrombus and Spontaneous Contrast on Transesophageal Echocardiography in Patients with Atrial Fibrillation. J Cardiovasc Electrophysiol 2013;24:1104-9.

99. Tischer TS, Schneider R, Lauschke J, Diedrich D, Kundt G, Bänsch D. Prevalence of atrial fibrillation and the HATCH score: Intensified monitoring of patients with high HATCH score. Herz 2015;40:803-8.

100. Kochhaeuser S, Dechering D, Trought K, Hache P, Haig-Carter T, Khaykin Y, et al. Progression of Paroxysmal to Persistent Af in Patients Awaiting AF Ablation. Circulation 2015;132:A14870-A14870.

101. Packer DL, Kowal RC, Wheelan KR, Irwin JM, Champagne J, Guerra PG, et al. Cryoballoon ablation of pulmonary veins for paroxysmal atrial fibrillation: first results of the North American Arctic Front (STOP AF) pivotal trial. J Am Coll Cardiol 2013;61:1713-23.

102. Lim HS, Denis A, Middeldorp ME, Lau DH, Mahajan R, Derval N, et al. Persistent atrial fibrillation from the onset: a specific subgroup of patients with biatrial substrate involvement and poorer clinical outcome. JACC Clin Electrophysiol 2016;2:129-39.

103. Goette A, Kalman JM, Aguinaga L, Akar J, Cabrera JA, Chen SA, et al. EHRA/HRS/APHRS/SOLAECE expert consensus on atrial cardiomyopathies: definition, characterization, and clinical implication. Europace 2016; euw161. 


\section{Figure legends}

Figure 1. Summary of the future directions towards an improved risk stratification system for predicting morbidity and mortality in AF 